\title{
The Biodynamic Microscope: Doppler Imaging inside Living 3D Biological Tissues.
}

\author{
David Nolte ${ }^{1,3}$, John Turek ${ }^{2,3}$ and Ran $\mathrm{An}^{3}$. \\ 1. Dept. of Physics, Purdue University, West Lafayette, IN USA 47907. \\ 2. Dept. of Basic Medical Sciences, Purdue University, West Lafayette, IN USA 47907. \\ 3. Animated Dynamics Inc. West Lafayette, IN USA 47906.
}

Biodynamic imaging is a new form of microscopy that combines coherent laser imaging with digital holography to extract Doppler images up to $1 \mathrm{~mm}$ deep inside living tissue [1]. Intracellular biophysical processes that are captured by biodynamic imaging include exo- and endocytosis, mitochondrial activity, nuclear morphology, cytoskeletal remodeling, cellular integrity, cell proliferation and mitosis, as well as necrosis and induced apoptosis, among others. Biodynamic microscopy is label-free, relying on Doppler scattering inside living tissue to provide image contrast. Biodynamic microscopy is fully $3 \mathrm{D}$, relying on low-coherence laser ranging techniques to extract three dimensional images. Biodynamic microscopy is non-invasive, using low intensity LED illumination. Because motion is ubiquitous in all living samples, biodynamic imaging is a general new form of microscopy that can be applied in any situation involving living 3D tissue or samples.

The number of applications of biodynamic microscopy is growing rapidly. This presentation provides an overview of a wide range of recent studies using biodynamic imaging. These are: 1) 3D tissue growth monitoring; 2) oocyte and embryo assessment for IVF applications; 3) chemotherapy sensitivity assays for personalized cancer care; and 4) phenotypic profiling for pharmaceutical drug discovery and development.

Three-dimensional Tissue Growth Monitoring: Three-dimensional tissue growth is a new market that is growing rapidly with the realization that conventional 2D cell culture fails to capture important threedimensional microenvironmental effects. Biodynamic imaging assesses differences in different approaches to 3D cell culture growth, such as bioreactors, hanging drops and nonadhesive plates. The use of biodynamic imaging to measure the response to applied pharmaceuticals of tissues, grown with these different methods, uncovered important differences that can relate to the downstream success rate for drug development.

IVF Applications: The success of assisted reproductive technologies relies on accurate assessment of reproductive viability at successive stages of development for oocytes and embryos. Biodynamic imaging measures physiologically-relevant dynamics and may provide an objective approach to oocyte and embryo assessment. The changes in intracellular activity during cumulus-oocyte complex maturation, before and after in vitro fertilization, and the subsequent development of the zygote and blastocyst provide a new approach to the assessment of pre-implant candidates.

Personalized Cancer Care: Intracellular motions encompass a diverse range of intracellular functions that provide fundamentally new biomarkers of the phenotypic response of living tissue to therapy. These dynamic biomarkers can be used to predict therapeutic efficacy for the treatment of disease in preclinical and clinical trials. A preclinical trial has been completed [2] for canine non-Hodgkins lymphoma using biodynamic imaging to predict patient outcome under doxorubicin therapy. The biodynamic assay took only 24 hours to complete, while the clinical outcome was often unknown for 
one to several months. The therapeutic efficacy of doxorubicin was predicted by BDI with $90 \%$ accuracy. Based on this success, biodynamic imaging is entering human pilot trials in ovarian, pancreatic, and esophageal cancer. Biodynamic imaging is a radically different approach to the prediction of therapeutic efficacy for personalized medicine with higher accuracy and more biological relevance than standard cell-based assays.

Drug Discovery: The existence of phenotypic differences in the drug responses of three-dimensional tissues relative to two-dimensional cell culture is a concern in high-content drug screening. The information content of biodynamic imaging for drug discovery applications is displayed through tissue dynamics spectroscopy (TDS), which captures and displays the changes in the Doppler signatures from intracellular constituents in response to applied compounds. Here we present the comparison of TDS against morphological image analysis of two-dimensional cell culture. There are significant $2 \mathrm{D}$ versus $3 \mathrm{D}$ phenotypic differences exhibited by $25 \%$ of the drugs/cell-lines which could relate to therapeutic efficacy and toxicity in 3D that are missed by 2D screens [3].

\section{References:}

[1] D. D. Nolte, R. An, J. Turek, and K. Jeong, "Holographic tissue dynamics spectroscopy," Journal of Biomedical Optics, vol. 16, pp. 087004-13, Aug 2011.

[2] M. R. Custead, J. J. Turek, R. An, D. D. Nolte, and M. O. Childress, "Use of biodynamic imaging to predict treatment outcome in a spontaneous canine model of non-Hodgkin's lymphoma," in preparation for submission to Cancer Research, 2015.

[3] R. An, D. Merrill, L. Avramova, J. Sturgis, M. Tsiper, J. P. Robinson, J. Turek, and D. D. Nolte, "Phenotypic Profiling of Raf Inhibitors and Mitochondrial Toxicity in 3D Tissue Using Biodynamic Imaging," Journal Of Biomolecular Screening, vol. 19, pp. 526-537, Apr 2014.

[4] The authors acknowledge funding from NSF1263753-CBET and NIH NIBIB 1R01EB016582-01.

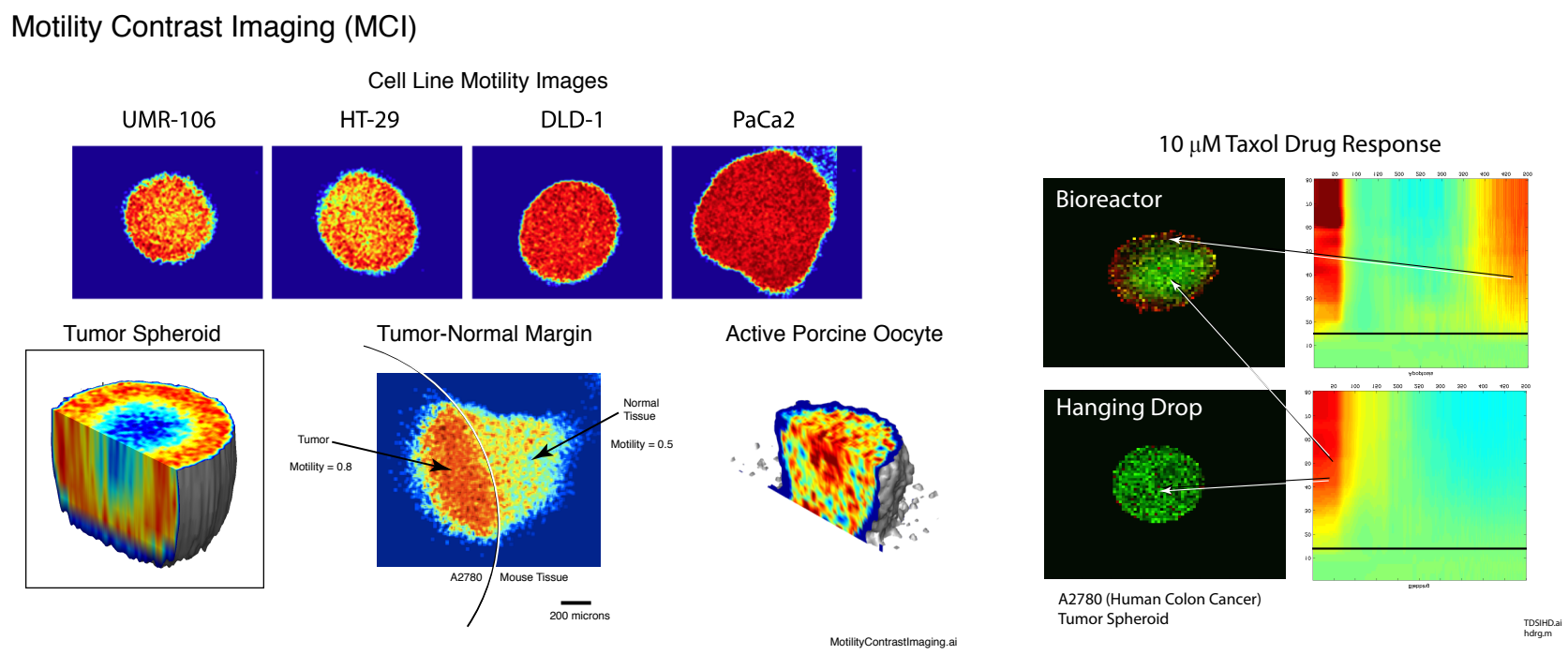

Figure 1. (Left) Examples of biodynamic images of 3D tumor spheroids, with a three dimensional reconstruction, a tumor margin and an oocyte. (Right) Tissue response to Taxol depends on growth technique (bioreactor vs. hanging drop). Tissue dynamics spectroscopy (TDS) is used for hyperspectral imaging. 